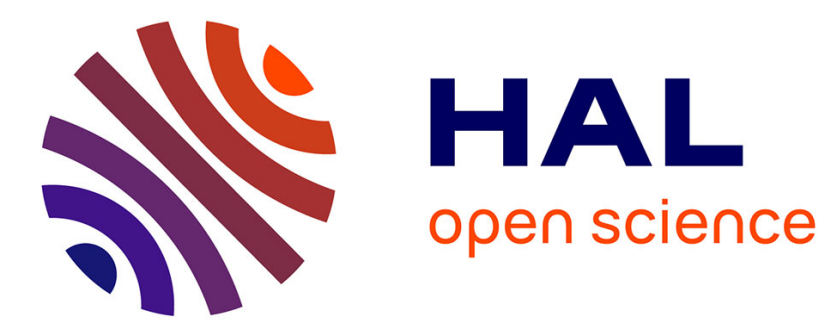

\title{
Effect of Object Width on Muscle and Joint Forces During Thumb-Index Finger Grasping
}

Laurent Vigouroux, Mathieu Domalain, Eric Berton

\section{To cite this version:}

Laurent Vigouroux, Mathieu Domalain, Eric Berton. Effect of Object Width on Muscle and Joint Forces During Thumb-Index Finger Grasping. Journal of Applied Biomechanics, 2011. hal-03389930

\section{HAL Id: hal-03389930 \\ https://hal.science/hal-03389930}

Submitted on 25 Oct 2021

HAL is a multi-disciplinary open access archive for the deposit and dissemination of scientific research documents, whether they are published or not. The documents may come from teaching and research institutions in France or abroad, or from public or private research centers.
L'archive ouverte pluridisciplinaire $\mathbf{H A L}$, est destinée au dépôt et à la diffusion de documents scientifiques de niveau recherche, publiés ou non, émanant des établissements d'enseignement et de recherche français ou étrangers, des laboratoires publics ou privés. 
(3366 words)

\section{EFFECT OF OBJECT WIDTH ON MUSCLE AND JOINT FORCES DURING THUMB-INDEX FINGER GRASPING}

Laurent VIGOUROUX $^{1}$; Mathieu DOMALAIN ${ }^{1}$; Eric BERTON ${ }^{1}$

${ }^{1}$ Movement Sciences Institute, Etienne Jules Marey, UMR 6233, Mediterranean University, Marseille, France.

Corresponding author:

Laurent VIGOUROUX

Address: $\quad$ U.M.R. 6233 Institut des Sciences du Mouvement, Faculté des Sciences du Sport, Case postale 910 , 163, avenue de Luminy F-13288 Marseille Cedex 09 France

Phone: $\quad+33(0) 491170422$

Fax: $\quad+33(0) 491172252$

Email: $\quad$ laurent.vigouroux@univmed.fr

Keywords: modeling, hand, pinch grip, object ergonomic, joint force, muscle force 


\section{Abstract (196 words):}

The objective of this study was to identify the impact of modifying the object width on tendon tensions and joint forces while gripping objects. The experimental protocol consisted to maintain horizontally five objects of different widths $(3.5,4.5,5.5,6.5$ and $7.5 \mathrm{~cm})$ with a thumb-index finger grip. Subjects were required to grasp spontaneously the object without any consign of applied fingertip force (GF). A biomechanical model of thumb-index finger pinch was developed to estimate tendon tensions and joint forces. This model included electromyography, fingertip force and kinematics data as inputs. The finger joint postures and the GF varied across the object widths. The estimated muscle forces also varied significantly according to the object width. Interestingly, we observed that the tendon tension/GF ratios of major flexors muscles remain particularly stable with respect to the width while other muscles ratios differed largely. This may argue for a control strategy in which the actions of flexors were preserved in spite of change in joint postures. The estimated joint forces tended to increase with object width and increased in the distal-proximal sense. Overall, these results are of importance for the ergonomic design of hand held objects and clinical problematic. 


\section{Introduction}

Grasping objects or tools is a predominant daily life activity such as a loss of this function is recognized as a major handicap (Armstrong \& Chaffin1978; Engelberg, 1988; Meagher, 1987; Wainstein \& Nailor, 2006). Obviously, the characteristics of the object grasped (width, load, shape) are predominant factors influencing the occurrence of pathologies. Particularly, the object width modifies the joint postures and so changes the muscle length, the muscle moment arms and finally, the muscle coordination what may lead to more risked and/or fatiguing conditions (Chao et al., 1989; Cooney \& Chao, 1977; Harding et al., 1993).

Many ergonomic studies have focused on the determination of the optimal object characteristics by selecting the width which maximizes the maximal grip force (MGF). Those studies typically report an inverted U-shape relationship, the optimal grip width varying around $5 \mathrm{~cm}$ depending on the posture adopted, the number of fingers involved and the shape of the object (Blackwell et al., 1999; Dempsey \& Ayoub, 1996; Fathallah et al., 1991; Fransson \& Winkel, 1991). From an ergonomic point of view, submaximal grip forces have to be taken into account for pathology prevention since during daily life objects are often manipulated with less intensity than $100 \%$ of MGF. Some other studies focused on the motor control of the external force spontaneously exerted ('grip force' GF) to hold an object (Westling \& Johansson, 1984). Few studies interested in the effect of width on GF. Interestingly, in some conditions the width which minimizes GF differs from the one which maximise MGF arguing for the including of GF concomitantly to MGF into the analysis (Domalain et al., 2008).

Even if these studies contribute to the understanding of grip tasks and to the object ergonomics, it remains crucial to understand how external forces (MGF and GF) are 
transmitted into internal forces exerted on muscles, tendons and joints which are the structures directly implicated in the pathologies. Indeed, because of finger postures and muscle coordination reorganization, a higher external force does not necessarily traduce higher internal forces and the other way round. As direct measurement of these variables is not possible, some biomechanical models of hand and fingers were developed: Cooney \& Chao (1977) and Chao et al., (1976) used this type of model to estimate muscle forces exerted while gripping an object with two fingers. Sancho-Bru et al. (2001) validated a biomechanical model for power grip with different size but did not include the thumb into the analysis. The most developed models used both mechanical data (external force, kinematics) and physiological data (electromyography, EMG) to estimate physiologically realistic muscle forces (Valero-Cuevas et al., 1998; Vigouroux et al., 2007).

In spite of this modeling possibility, nothing is known about the influence of the object width on muscle/joint forces while gripping an object. This leads to ergonomic conceptions which are currently focused on the external forces results only, without quantifying their impact on the anatomical structures. The objective of this study was thus to investigate the effect of object width on muscle forces and the joint forces. As a first step, the spontaneous grip of object with an index/thumb fingers pinch grip was studied. A biomechanical model of index/thumb was developed to determine internal forces. We hypothesized that even with the possible reorganization of joint postures, object width would impact significantly muscle and joint forces.

\section{Methods}

\section{Subjects}

Ten right-handed subjects participated in this study (age: $27.4 \pm 3.9$ years; height: $180.2 \pm 4.0$ $\mathrm{cm}$; body mass: $76.6 \pm 5.6 \mathrm{~kg}$; hand size: $19.6 \pm 0.6 \mathrm{~cm}$; Mean \pm SD). None of the participant 
had any history of trauma affecting the upper limbs. All subjects signed an informed consent approved by the University guideline.

\section{Experimental set-up and procedure}

The participants were seated in a chair with the right elbow and the palm of the hand supported by two clamps so that no effort was made by the muscles to stabilize the hand and wrist. The shoulder was placed at $45^{\circ}$ of flexion and abduction. The elbow was fixed at $80^{\circ}$ of flexion (full extension being $\left.0^{\circ}\right)$. The wrist was in neutral position $\left(0^{\circ}\right.$ flexion and $0^{\circ}$ of radioulnar deviation) with forearm pronated. The thumb and index finger were positioned next to the clamp so that they could move freely. The task consisted in grasping objects of five different widths $(3.5,4.5,5.5,6.5$ and $7.5 \mathrm{~cm})$. The weight of the five objects was equal $(0.5 \mathrm{~kg})$. The participants were instructed to maintain the object horizontally between their index and thumb fingers during 6 seconds. Subjects were unaware of the characteristics of the objects and no particular consign on how to grip was given. Also, no verbal or visual feedback was given. Positioning of the object was recorded using three reflective markers. Surfaces at digitobject interface consisted of $8 \mathrm{~mm}$ diameter discs covered by fine grain sandpaper ( 80 grains $/ \mathrm{cm}^{2}$ ) and participants'digits were cleaned with alcohol before the experiment. Three trials were performed for each size object. One minute rest periods were provided between each trial to avoid any effect of fatigue. The order of the conditions was randomized to avoid any order effect.

\section{Fingertip force}

A six-axial force sensor (Nano-25, ATI Industrial Automation, Garner, NC) was embedded in the objects in order to record the grip force and the moments applied to the object. The force and moments signals were recorded at $100 \mathrm{~Hz}$ using National Instrument acquisition products (NI-PCI 6220, USA and a customized Labview program). 
Surface EMG of five muscles (abductor pollicis brevis APB; abductor pollicis longus APL; Extensor digitorum communis, EDC; flexor pollicis longus FPL, First Radial Interosseous RI) were recorded at $2 \mathrm{kHz}$ with a BIOPAC system (bandpass from $10 \mathrm{~Hz}$ to $5 \mathrm{kHz}$; amplification to $3 \mathrm{db}$; common mode rejection ratio: $>90 \mathrm{~dB}$ ) and the associated Acqknowledge 3.8.1 software. Skin of the subjects was abraded and cleaned before electrodes placement. $10 \mathrm{~mm}$ width electrodes (EL503, Cerom) were used to record APL, EDC, FPL. As APB and RI were small sized muscles, $4 \mathrm{~mm}$ Electrodes (EL254S, Cerom) were used to record their activities. Placements of electrodes were made according to Basmajian and Blumstein (1989) and were adjusted by palpation. Good positioning of the electrodes was ensured by testing functional movements corresponding to the recorded muscle function. Concerning the FPL and APL muscles, the positioning of the electrodes was made closed to the wrist at the beginning of the muscle belly. At this location, FPL and APL are not covered by other surface muscles. EMG signals were filtered off-line using a zero-lag Butterworth filter (order 4, bandpass from 20 to $400 \mathrm{~Hz}$ ). Force and EMG acquisition tools were synchronized thanks to a rising edge trigger.

\section{Kinematic}

The 3D positioning of each thumb and index segment was recorded by a six cameras system (Vicon 624 Motion System, Oxford Metrics, England). Three spherical micro-reflective markers ( $4 \mathrm{~mm}$ diameter) were fixed on each segment using T-shape supports. Three markers, placed on the metacarpal bones were used to define the dorsal hand plane reference system ( $\mathbb{R}_{\text {dhp }}$ ). Joint angles were computed from the 3D positioning of the segments for both thumb and index finger models. The thumb and index were both considered as four segments articulated by three joints. The interphalangeal joint of the thumb (IP), the distal and the proximal interphalangeal joints of the index (DIP, PIP) were considered as 1 degree of 
freedom $(\mathrm{DoF})$ in flexion/extension. The metacarpophalangeal joints of the thumb and the index (MP and MCP), the trapeziometacarpal joint of the thumb (TMC) were considered as 2 DoFs in flexion/extension and in adduction/abduction.

The angle of IP, MP, DIP, PIP and MCP were defined as rotation between distal and proximal segments using reference systems placed on the metacarpal bones, the proximal phalanges and the distal phalanges. The TMC joint angles were defined as the rotation between thumb metacarpal and the trapezium bone reference system $\left(R_{\mathrm{t}}\right)$. The positioning of $\mathbb{R}_{\mathrm{t}}$ was determined from Cooney et al. (1981) who reported that $R_{\mathrm{t}}$ is rotated by $46^{\circ}$ of flexion, $35^{\circ}$ of abduction and $82^{\circ}$ of supination with respect to $\mathcal{R}_{\text {dhp. }}$. Angles were extracted from the rotation matrix using the Z, Y, X, Euler's sequence (i.e flexion, abduction, supination) with fixed axes situated on the proximal segment to follow the method of Cooney et al. (1981). Abduction and flexion have positive values.

\section{Data analysis}

Averaged fingertip forces and finger postures were calculated within a $750 \mathrm{~ms}$ window centred on the force plateau. Joint moments were then computed for input of biomechanical model. Within this time interval, muscle excitation levels for each muscle ( $\mathrm{e}_{\mathrm{m}}$ with $m=\mathrm{APB}$, APL, EDC, FPL, RI) were computed:

$\mathrm{e}_{\mathrm{m}}=\frac{\mathrm{RMS}_{\mathrm{m}}}{\mathrm{RMS}_{\mathrm{m} \max }}$

where $\mathrm{RMS}_{\mathrm{m}}$ was the EMG root mean square value computed for each test (Basmajian \& De Luca, 1985). $\mathrm{RMS}_{\mathrm{m} \max }$ corresponded to the largest root mean square value recorded during additional maximal voluntary tasks performed in the same posture in various external force directions (flexion, extension, adduction and abduction of the thumb and index fingers). 


\section{Biomechanical finger model}

The index and thumb muscolo-skeletal systems were modeled as previously described (Chao et al., 1989; Vigouroux et al., 2008; Vigouroux et al., 2009) and were used to compute muscle forces. The index was mobilised by 7 muscles (flexor digitorum profundus FDP, flexor digitorum superficialis FDS, lumbrical LU, ulnar interosseus UI, RI, EDC, extensor digitorum indicis EDI) and the thumb by 10 muscles (FPL, flexor pollicis brevis FPB, opponents pollicis $\mathrm{OPP}, \mathrm{APB}$, adductor pollicis oblique head ADPo, adductor pollicis tranverse head ADPt, APL, extensor pollicis longus EPL, extensor pollicis brevis EPB). The model results in 9 equilibrium moment equations and 16 unknown muscle forces resumed as follow:

$[\mathrm{R}] \cdot\{\mathrm{T}\}+\{\mathrm{L}\}+\{\mathrm{F}\}=\{0\}$

where the $9 \times 16$ matrix $[R]$ is the moment arms matrix obtained from moment arms of muscles and from the coefficients associated with the extensor mechanism (Vigouroux et al., 2007; Vigouroux et al., 2009). The muscle moment arms were estimated from the finger joint angles for each finger using the results of Chao et al. (1989). $\{\mathrm{T}\}$ is the 16-elements vector containing the unknown muscle forces. $\{\mathrm{L}\}$ is the vector containing the passive moment over MCP (Sancho-Bru et al., 2001) and TMC (see part below) due to the ligament and passive joint structures. $\{\mathrm{F}\}$ is the 9-elements vector representing moments of external force at each degree of freedom of the index and thumb fingers. $\{\mathrm{F}\}$ was computed from the joint angles, the external fingertip forces and the segment lengths. The under-determined problem was solved with an "optimization constrained by EMG" process (Vigouroux et al., 2007). This optimization process used a muscle stress criterion to determine an optimal set of muscle forces and included additional inequality constraints which account for the $\mathrm{e}_{\mathrm{m}}$ of the muscles recorded by EMG. Results of the optimization process were the muscle forces. Muscles forces 
were normalized by the external fingertip force (muscle force-external force ratio) in order to evaluate the action of muscles in regards to fingertip force. Using the nine equations of force equilibrium the joint forces were computed for each joint. For this computing, all muscle force intensities were considered as well as their respective unit force direction vector. This was performed by using the anthropometric data of Chao et al. (1989) which described the positioning of the tendons at each joint according to pulley and bones characteristics. The intensity of each joint force was computed by considering both shear and normal components.

\section{Passive constraints}

During pulp pinch grip, the TMC joint is naturally positioned near its end range of motion to provide the necessary stability at this joint (Napier, 1956). This leads to an important participation of soft tissues (skin, ligaments, tendons, etc.) in the equilibrium of joint forces. Thus, the results of Domalain et al. (2010) were used to determine the passive moment at the TMC and were included into the Eq. $1(\{\mathrm{~L}\})$. The model of Domalain et al. (2010) is a generic model which could be sensible to kinematic errors and individual properties. As the amplitude of external force moments in the current gripping tasks were weak in regards to maximal voluntary forces, the estimation error could thus represent a larger proportion. For some subjects this resulted in TMC passive moments slightly superior than external force moment. In this case, we decided to limit the passive joint moment to $90 \%$ of the external joint moment. The passive constraints of IP, MP, DIP and PIP joints were neglected as these joints were mobilized far from their end range of motion.

\section{Statistical analysis}

Results of each subject were averaged across the three trials. Normality of the results was verified. Descriptive statistics are mean and standard deviation $( \pm \mathrm{SD})$. Repeated-measure 
Anovas were used to identify the effect of object size on external fingertip force, joint angles, muscle forces, muscle force-external force ratio and joint forces. A level of $p<0.05$ was considered as significant.

\section{Results}

GF spontaneously applied on the object (Fig. 1) were significantly different with respect to the object width $(\mathrm{F}(4,36)=4.0 ; p<0.05)$. GF amounted to $6.2 \pm 1.8 \mathrm{~N}$ for a $3.5 \mathrm{~cm}$ object width, $5.7 \pm 1.2 \mathrm{~N}$ for $4.5 \mathrm{~cm}, 4.8 \pm 1.7$ for $5.5 \mathrm{~cm}, 5.5 \pm 1.0 \mathrm{~N}$ for $6.5 \mathrm{~cm}$ and $6.0 \pm 1.4 \mathrm{~N}$ for $7.5 \mathrm{~cm}$.

In thumb, IP flexion $(\mathrm{F}(4,36)=1.6 ; p>0.05)$, MP flexion $(\mathrm{F}(4,36)=1.7 ; p>0.05)$, MP Abduction $(\mathrm{F}(4,36)=1.6 ; p>0.05)$ and $\mathrm{TMC}$ abduction $(\mathrm{F}(4,36)=0.22 ; p>0.05)$ did not varied significantly with respect to the object width. IP mean flexion was $13.3^{\circ} \pm 12.5$, MP flexion averaged $3.5^{\circ} \pm 9.1$. MP and TMC abduction averaged $15.9^{\circ} \pm 8.7$ and $-8.2 \pm 8.6$ respectively. A significant effect $(\mathrm{F}(4,36)=18.3 ; p<0.05)$ was observed for TMC flexion which extend slightly and progressively to $19.1^{\circ} \pm 4.8,17.1^{\circ} \pm 4.3,14.7 \pm 6.1^{\circ}, 12.5^{\circ} \pm 5.8,11.1^{\circ} \pm 4.9$ for $3.5,4.5,5.5$, 6.5 and $7.5 \mathrm{~cm}$ respectively. Index posture changes significantly with object width. DIP flexion $(\mathrm{F}(4,36)=6.9 ; p<0.05)$ varied from $20.9 \pm 11.8^{\circ}$ with $3.5 \mathrm{~cm}$, to $29.6 \pm 9.0^{\circ}$ with $7.5 \mathrm{~cm}$. PIP flexion also varied significantly $(\mathrm{F}(4,36)=6.5 ; p<0.05)$ from $22.1 \pm 14.0^{\circ}$ at $3.5 \mathrm{~cm}$, $17.2 \pm 11.5^{\circ}$ at $4.5 \mathrm{~cm}, 12.6 \pm 13.6^{\circ}$ at $5.5 \mathrm{~cm}, 11.2 \pm 10.3^{\circ}$ at $6.5 \mathrm{~cm}$ and $13.8 \pm 11.2^{\circ}$ at $7.5 \mathrm{~cm}$. $\mathrm{MCP}$ abduction did not varied significantly $(\mathrm{F}(4,36)=1.91 ; p>0.05)$ and averaged $-4.0^{\circ} \pm 9.4$. $\mathrm{MCP}$ extend progressively $(\mathrm{F}(4,36)=13.0 ; p<0.05)$ from $55.6 \pm 12.6^{\circ}$ at $3.5 \mathrm{~cm}$ to $38.6 \pm 14.0^{\circ}$ at $7.5 \mathrm{~cm}$.

The figure 2 and 3 represents the muscle forces of index muscles and thumb muscles for a $5 \mathrm{~cm}$ width object. The table 1 displays the mean muscle forces observed in each muscle with respect to the object width. There is a significant effect of object width for FDP $(F(4,36)=2.7$; $p=0.05), \operatorname{FDS}(\mathrm{F}(4,36)=7.3 ; p<0.05), \mathrm{UI}(\mathrm{F}(4,36)=4.4 ; p<0.05), \operatorname{EDC}(\mathrm{F}(4,36)=2.6 ; p=0.05)$, 
and $\operatorname{EDI}(\mathrm{F}(4,36)=3.5 ; p<0.05)$. FDP, FDS and RI developed the highest force intensities for the index finger. Concerning thumb muscles, a significant effect of object width on muscle force was observed for $\operatorname{ADPt}(\mathrm{F}(4,36)=4.0 ; p<0.05), \operatorname{ADPo}(\mathrm{F}(4,36)=3.6 ; p<0.05), \mathrm{APB}$ $(\mathrm{F}(4,36)=4.8 ; p<0.05), \mathrm{OPP}(\mathrm{F}(4,36)=3.1 ; p<0.05), \mathrm{EPL}(\mathrm{F}(4,36)=4.2 ; p<0.05)$ and $\mathrm{EPB}$ $(\mathrm{F}(4,36)=8.3 ; p<0.05)$. FPL, FPB and APL presented no significant difference $(p>0.05)$ with respect to object width. The highest muscle forces were observed in FPL, OPP, ADPt and EPL muscles for thumb.

The figure 4 represents the muscle force-external force ratio with respect to the object width.

The effect of object width was significant for $\operatorname{FDS}(\mathrm{F}(4,36)=8.2 ; p<0.05), \mathrm{UI}(\mathrm{F}(4,36)=4.5$; $p<0.05), \operatorname{EDC}(\mathrm{F}(4,36)=3.1 ; p<0.05)$, and $\operatorname{EDI}(\mathrm{F}(4,36)=3.7 ; p<0.05)$. For EDI, EDC and FDS the ratios increase with the increase in object width while for UI, we observed a decrease. No significant effect of object width was observed for FDP, LU and RI muscle. Concerning the thumb, the effect of object width on muscle force-external force ratio was significant for $\operatorname{ADPo}(\mathrm{F}(4,36)=4.0 ; p<0.05), \operatorname{ADPt}(\mathrm{F}(4,36)=4.0 ; p<0.05), \operatorname{APB}(\mathrm{F}(4,36)=2.8 ; p<0.05), \mathrm{OPP}$ $(\mathrm{F}(4,36)=2.75 ; p<0.05), \operatorname{EPL}(\mathrm{F}(4,36)=7.0 ; p<0.05), \operatorname{EPB}(\mathrm{F}(4,36)=7.3 ; \mathrm{p}<0.05) . \mathrm{EPL}$ and EPB muscle force-external force ratio increased with object width, APB decreased progressively while OPP, ADPt and ADPo did not showed a simple evolution. No significant effect appeared for FPL, FPB and APL.

Figure 5 presents the joint forces with respect to the object width. A significant effect of object width was observed in PIP joint $(F(4,36)=4.0 ; p<0.05)$. No significant effect was observed in the TMC $(\mathrm{F}(4,36)=1.9 ; p>0.12)$ although a significant tendency was observed for DIP joint $(\mathrm{F}(4,36)=2.2 ; p=0.09), \operatorname{MCP}(\mathrm{F}(4,36)=2.47 ; p=0.06), \operatorname{IP}(\mathrm{F}(4,36)=0.3 ; p=0.8), \mathrm{MP}$ $(\mathrm{F}(4,36)=2.1 ; p=0.09)$. Whatever the width and the finger, the finger joint force increased 
from IP and DIP to MP and MCP. Mean joint forces ranged from 13.9N for the distal joints (IP and DIP) to $76.5 \mathrm{~N}$ for the proximal joints (MCP, TMC).

\section{Discussion}

This study aimed to explore the effect of the object width on the muscle forces and joint forces while gripping spontaneously an object. The understanding of the internal constraints caused by changes in object width could have a great impact first for the ergonomic field, to shape objects and tools which prevent pathology and fatigue, and second for the clinical field, to improve surgical and rehabilitation programs.

As previously observed, the index finger adapted its posture (DIP flexion increased while PIP and MCP extended) to the object width while the thumb postures changes in smallest proportion (only TMC flexion slightly decreased with object width). The grip force used to maintain the object was different with respect to the object width. It was well defined into the literature that people use more force than necessary to grip and hold an object what is generally defined as a "security margin", (Westling \& Johansson, 1984). The current results of grip force demonstrated that the security margin varied with respect to the object width in spite of the similar weight, contact surfaces and friction characteristics. This phenomenon was already observed into the literature but has still remained unexplained (Domalain et al. 2008). In our study we showed an inverted U-shape relationship centered at $5.5 \mathrm{~cm}$ width which differed from Domalain et al. (2008) who showed an increase from $3.5 \mathrm{~cm}$ to $9.5 \mathrm{~cm}$. This might be explained by the fact that in the current study we didn't test a $9.5 \mathrm{~cm}$ width object where the greatest differences were observed in Domalain et al. (2008). Moreover, we tested smaller load $(0.5 \mathrm{~kg})$ than in Domalain et al. $(2008)(1 \mathrm{~kg}, 1.5 \mathrm{~kg}$ and $2.25 \mathrm{~kg})$ who observed the largest effect with the largest load. Globally, this result showed that the central nervous system (CNS) did not consider the intensity of GF and the value of security margin as an 
absolute objective. The parameters the GF was adjusted to still remain unknown and are probably numerous. However, the results of muscle and tendon forces indicate us that they may be considered into the reflection.

The change in grip force and in finger postures resulted in significant changes in all muscles excepted in EPL, APL, FPB for the thumb and RI, LI for the index finger. Similar effects were observed in the muscle force/external force ratios excepted for the FDP muscle which remained constant. When looking globally at these changes, one can observe an increase of extensor muscle force-external force ratio in both index (EDC and EDI) and thumb (EPL and EPB) with the increased width. This phenomenon could be associated to a strategy which would consist in extending the posture of MCP and TMC to account for the increase of object width. It was also observed that UI and APB decreased progressively their forces with the increase of object width. Then the muscles acting on the opposite side of the finger-object contact surface were less and less solicited. Even if extensor muscles and adductor/abductor muscles acted as antagonist in the studied tasks, we hypothesized that their actions were crucial to stabilize the joints and to position the joints in favorable conditions. The purpose of such a strategy could be to first adapt a favorable joint posture in order that the finger tip was placed ideally on the object and second that joint angles were favourable for the flexor muscle action. This hypothesis is enforced by the fact that FPL and FDP were solicited in a constant manner (stable muscle force-external force ratio) in spite of object width changes. This suggests that angles, muscle coordination may be selected in order to keep these ratios constant. FPL and FDP were indeed the only flexors acting on the distal joints. These muscles were thus crucial for the success of the task. Then adapting the action of other muscles not directly implicated in the task to preserve the action of FPL and FDP could be a strategy to success the task whatever the object width. 
Concerning the joint forces, no significant effect was observed except for the PIP joint. In spite of no significant effect, a tendency was observed in DIP, MP and MCP. The values of joint forces indeed increased progressively with the width but not significantly. It would be interesting to test larger widths $(8.5$ and $9.5 \mathrm{~cm})$ to confirm or infirm these tendencies.

Whatever the effect of object width, we observed a large increase of joint forces with the proximity of the joints. DIP and IP joint forces were inferior to IP an MP joint forces which are in turn inferior to MCP and TMC. This phenomenon could be explained by the action of the muscle. More muscles act on proximal joints and create thus more compressive forces than on distal joint crossed by fewer muscles. These considerations and the estimated values may be taken into account for pathologies analysis such arthritis.

The use of biomechanical models is the single methods available to provide quantitative data of internal forces. However, it should be noticed that the provided estimations of muscle and joint forces could be source of errors due to error in kinematic analysis, EMG recording and anthropometric data, under-determined problem (Valero-Cuevas et al., 2003; Vigouroux et al., 2009). The results of $0 \mathrm{~N}$ tension presented in LU and FPB muscles were probably witness of this kind of errors. The results and conclusions of this study thus should be considered by keeping in mind these limits. As a second limitation, it should be considered that the hand size of the tested subjects averaged $19.6 \pm 0.6 \mathrm{~cm}$. During grasping, the hand size of the subjects could modify the joint posture, the net joint moment, the lengths of bones and muscle moment arms. Consequently, it is not known if our conclusions still valid for subject with larger or smaller hands.

To resume this study detailed the effect of object width on external forces and internal forces. It was showed that the changes of external forces (following a U inverted relationship) were not necessarily followed by similar changes in muscle forces and joint forces. This suggested coordination strategy to preserve the action of the muscles directly implicated in the success 
of the task. From an ergonomics point of view, our results would suggest to limit the largest objects in order to preserve extensor muscles. This argument becomes crucial when considering that during grip (i.e. flexing) fatiguing exercise, extensor fatigued at the same rate than the flexor muscles (Quaine et al., 2003). Moreover, our results suggested that gripping an object with the fingertip may have a great impact on proximal joints due to muscle compression. Further researches are needed to compare this impact when grip force is applied at the level of proximal and middle phalanxes as during five digits grasping. 


\section{References}

An, K. N., Chao, E. Y., Cooney, I., W. P., \& Linscheid, R. L. (1979). Normative model of human hand for biomechanical analysis. Journal of Biomechanics, 12, 775-788

Armstrong, T., \& Chaffin, D.B. (1978). An investigation of the relationship between displacements of the finger and wrist joints and the extrinsic finger flexor tendons. Journal of Biomechanics 11, 119-128

Basmajian, J.V., \& De Luca, C.J. (1985). Muscles alive. Baltimore: Williams \& Wilkins

Blackwell, J. R., Kornatz, K. W., \& Heath, E. M. (1999). Effect of grip span on maximal grip force and fatigue of flexor digitorum superficialis. Applied Ergonomics, 30, 401-405

Challis, J.H. (1997). Producing physiologically realistic individual muscle force estimations by imposing constraints when using optimization techniques. Medical Engineering and Physics, 19, 253-61.

Chao, E., An, K.N., Cooney, W.P., \& Linscheid, R.L. (1989). Biomechanics of the Hand: A Basic Research Study: World Scientific Singapore

Chao, E.Y., Opgrande, J.D., \& Axmear, F.E. (1976). Three-dimensional force analysis of fingerjoints in selected isometric hand functions. Journal of Biomechanics, 9, 387-396

Cooney, W., \& Chao, E. (1977). Biomechanical analysis of static forces in the thumb during hand function. Journal of Bone and Joint Surgery, American volume, 59, 27-36

Cooney, W., Lucca, M., Chao, E., \& Linscheid, R. (1981). The kinesiology of the thumb trapeziometacarpal joint. Journal of Bone and Joint Surgery, American volume, 63, 1371-1381

Domalain, M., Vigouroux, L., Danion, F., Sevrez, V., \& Berton, E. (2008). Effect of object width on precision grip force and finger posture. Ergonomics, 51, 1441 - 1453 
Domalain, M., Vigouroux, L., \& Berton, E. (2010). Determination of passive moment - angle relationships at the trapeziometacarpal joint. Journal of Biomechanical Engineering. In press

Dempsey, P. G., \& Ayoub, M. M. (1996). The influence of gender, grasp type, pinch width and wrist position on sustained pinch strength. International Journal of Industrial Ergonomics, 17, 259-273

Engelberg, A. (1988), Guides to the evaluation of permanent impairment, American Medical Association

Fathallah, F. A., Kroemer, K. H. E. \& Waldron, R. L. (1991). A new finger strength pinch gage. International Journal of Industrial Ergonomics 7, 71-72

Fransson, C. \& Winkel, J. (1991). Hand strength: The influence of grip span and grip type. Ergonomics 34, 881-892

Harding, D., Brandt, K., \& Hillberry, B. (1993). Finger joint force minimization in pianists using optimization techniques. Journal of Biomechanics, 26, 1403-1412

Meagher, S. (1987). Tool design for prevention of hand and wrist injuries. American Journal of Hand and Joint Surgery, 12, 855-857

Napier, J. (1956). The prehensile movements of the human hand. Journal of Bone \& Joint Surgery, British Volume, 38, 902-913

Quaine, F., Vigouroux, L., \& Martin, L. (2003). Finger flexors fatigue in trained rockclimbers and untrained sedentary subjects. International Journal of Sport Medicine 24, $424-427$

Sancho-Bru, J. L., Perez-Gonzalez, A., Vergara-Monedero, M., \& Giurintano, D. (2001). A 3D dynamic model of human finger for studying free movements. Journal of Biomechanics, 34, 1491-1500 
Valero-Cuevas, F.J., Zajac, F.E., \& Burgar, C.G., (1998). Large index-fingertip forces are produced by subject-independent patterns of muscle excitation. Journal of Biomechanics 31, 693-703.

Valero-Cuevas, F. J., Johanson, M. E., \& Towles, J. D. (2003). Towards a realistic biomechanical model of the thumb: the choice of kinematic description may be more critical than the solution method or the variability/uncertainty of musculoskeletal parameters. Journal of Biomechanics, 36, 1019-1030

Vigouroux, L., Quaine, F., Labarre-Vila, A., Amarantini, D., \& Moutet, F. (2007). Using EMG data to constrain optimization procedure improves finger tendon tension estimations during static fingertip force production. Journal of Biomechanics, 40, $2846-2856$

Vigouroux, L., Quaine, F., Paclet, F., Colloud, F., \& Moutet, F. (2008). Middle and ring fingers are more exposed to pulley rupture than index and little during sport-climbing: A biomechanical explanation. Clinical Biomechanics, 23, 562 - 570

Vigouroux, L., Domalain, M., \& Berton, E. (2009). Comparison of tendon tensions estimated from two biomechanical models of the thumb. Journal of Biomechanics, 42, 1772-7

Wainstein, J.L., \& Nailor, T.E. (2006). Tendinitis and tendinosis of the elbow, wrist, and hands. Clinics in Occupational and Environmental Medicine, 5: 299-322

Westling, G., \& Johansson, R., 1984, Factors influencing the force control during precision grip. Experimental Brain Research, 53, 277-284 


\begin{tabular}{lccccc}
\hline \hline Muscle & $\mathbf{3 . 5}$ cm width & $\mathbf{4 . 5}$ cm width & $\mathbf{5 . 5}$ cm width & $\mathbf{6 . 5} \mathbf{~ c m}$ width & $\mathbf{7 . 5}$ cm width \\
\hline \hline FDP (*) & $16.9 \pm 6.7$ & $14.0 \pm 4.1$ & $13.5 \pm 7.9$ & $14.5 \pm 4.8$ & $18.0 \pm 9.6$ \\
FDS (*) & $9.6 \pm 4.4$ & $13.6 \pm 5.9$ & $11.9 \pm 7.0$ & $17.8 \pm 8.4$ & $17.6 \pm 5.7$ \\
LU & $0.1 \pm 0.02$ & $0.1 \pm 0.03$ & $0.01 \pm 0.03$ & $0.00 \pm 0.01$ & $0.0 \pm 0.0$ \\
RI & $17.5 \pm 7.9$ & $16.5 \pm 10.6$ & $14.0 \pm 7.8$ & $20.7 \pm 14.4$ & $20.3 \pm 15.1$ \\
UI (*) & $6.4 \pm 7.6$ & $5.2 \pm 7.7$ & $5.3 \pm 6.2$ & $3.3 \pm 7.5$ & $1.7 \pm 3.4$ \\
EDC (*) & $8.4 \pm 5.5$ & $8.5 \pm 5.2$ & $9.6 \pm 5.3$ & $12.6 \pm 7.6$ & $12.0 \pm 6.4$ \\
EDI (*) & $7.3 \pm 10.0$ & $9.0 \pm 8.8$ & $11.6 \pm 9.3$ & $16.2 \pm 12.3$ & $15.5 \pm 10.6$ \\
\hline \hline FPL & $10.3 \pm 5.4$ & $10.5 \pm 5.5$ & $9.5 \pm 5.0$ & $9.6 \pm 4.6$ & $10.6 \pm 5.0$ \\
FPB & $0.1 \pm 0.03$ & $0.0 \pm 0.0$ & $0.0 \pm 0.0$ & $0.0 \pm 0.0$ & $0.0 \pm 0.01$ \\
OPP (*) & $18.3 \pm 7.4$ & $15.7 \pm 6.8$ & $12.9 \pm 7.0$ & $15.0 \pm 6.5$ & $20.3 \pm 7.8$ \\
APB (*) & $6.8 \pm 2.8$ & $4.5 \pm 2.1$ & $3.3 \pm 1.6$ & $2.9 \pm 1.8$ & $3.2 \pm 1.9$ \\
ADPo (*) & $2.6 \pm 1.3$ & $2.7 \pm 1.3$ & $1.8 \pm 1.4$ & $2.7 \pm 1.3$ & $2.7 \pm 1.3$ \\
ADPt (*) & $18.7 \pm 9.5$ & $19.1 \pm 9.4$ & $13.1 \pm 10.1$ & $19.4 \pm 9.6$ & $19.3 \pm 9.2$ \\
APL & $9.5 \pm 3.8$ & $8.5 \pm 3.0$ & $8.9 \pm 3.1$ & $8.8 \pm 3.1$ & $9.5 \pm 3.4$ \\
EPL (*) & $13.5 \pm 6.6$ & $14.9 \pm 6.3$ & $15.3 \pm 6.3$ & $18.5 \pm 6.8$ & $20.2 \pm 8.4$ \\
EPB (*) & $0.3 \pm 0.9$ & $0.7 \pm 0.9$ & $1.4 \pm 0.9$ & $1.4 \pm 0.8$ & $1.5 \pm 0.9$ \\
\hline \hline
\end{tabular}

Mean tendon tensions $(\mathrm{N})$ across the ten subjects according to the object width. $\left({ }^{*}\right)$ indicates a 409 significant effect of object width $(p<0.05)$. First part of the table presents the index muscles 410 while the last nine lines presents the thumb muscles. 


\section{Figure legends}

412 Figure 1: Mean (SD) external forces (N) applied by the fingers on the object according to the object width. A significant effect of object width was observed on the force intensity.

414 Figure 2: Schematic representation of tendon forces applied by the extrinsic muscles acting on 415 index and thumb finger during a $5.5 \mathrm{~cm}$ width object.

416 Figure 3: Schematic representation of tendon forces applied by the intrinsic muscles acting on 417 index and thumb finger during a $5.5 \mathrm{~cm}$ width object.

418 Figure 4: Mean muscle force-external force ratio (u.a) observed in flexor muscles (A), extensor muscles (B), abductor muscles (C) and adductor muscles (D) according to object width. Thumb muscles were drawn with grey dashed lines and index muscles with black lines. To keep the figure clear, the SD was not drown. The variability could be however appreciated in Figure 1 and in Table 1 which represent the mean and SD of the two variables used to compute the muscle force-external force ratio.

Figure 5: Mean (SD) joint forces in index joints (upper figure) and thumb joints (figure below). The blocks represent the intensity of the joint forces computed from both shear and with a white triangle $(\Delta)$. 
$430 \quad$ Figure 1:

431

432

433

434

435

436

437

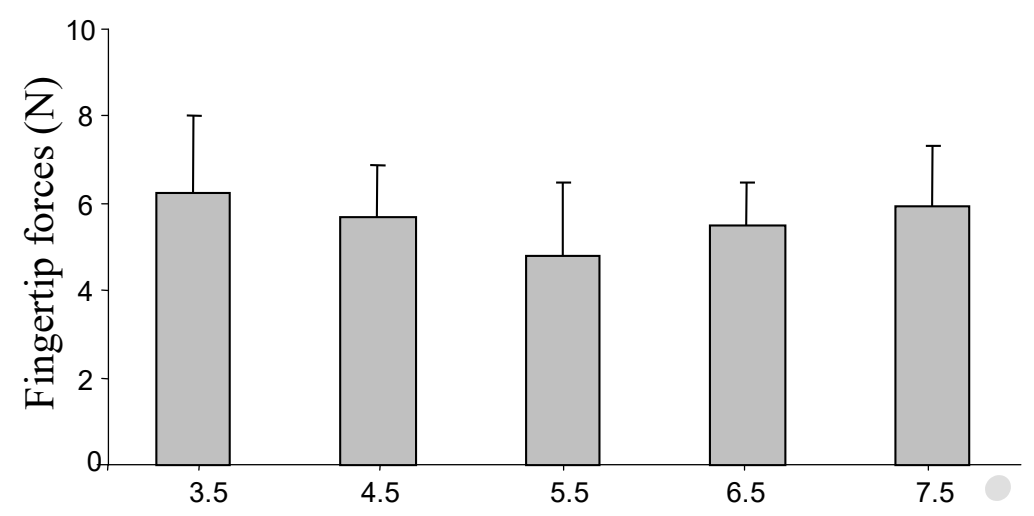

Object width $(\mathrm{cm})$

438

439 
$440 \quad$ Figure 2:

441

442

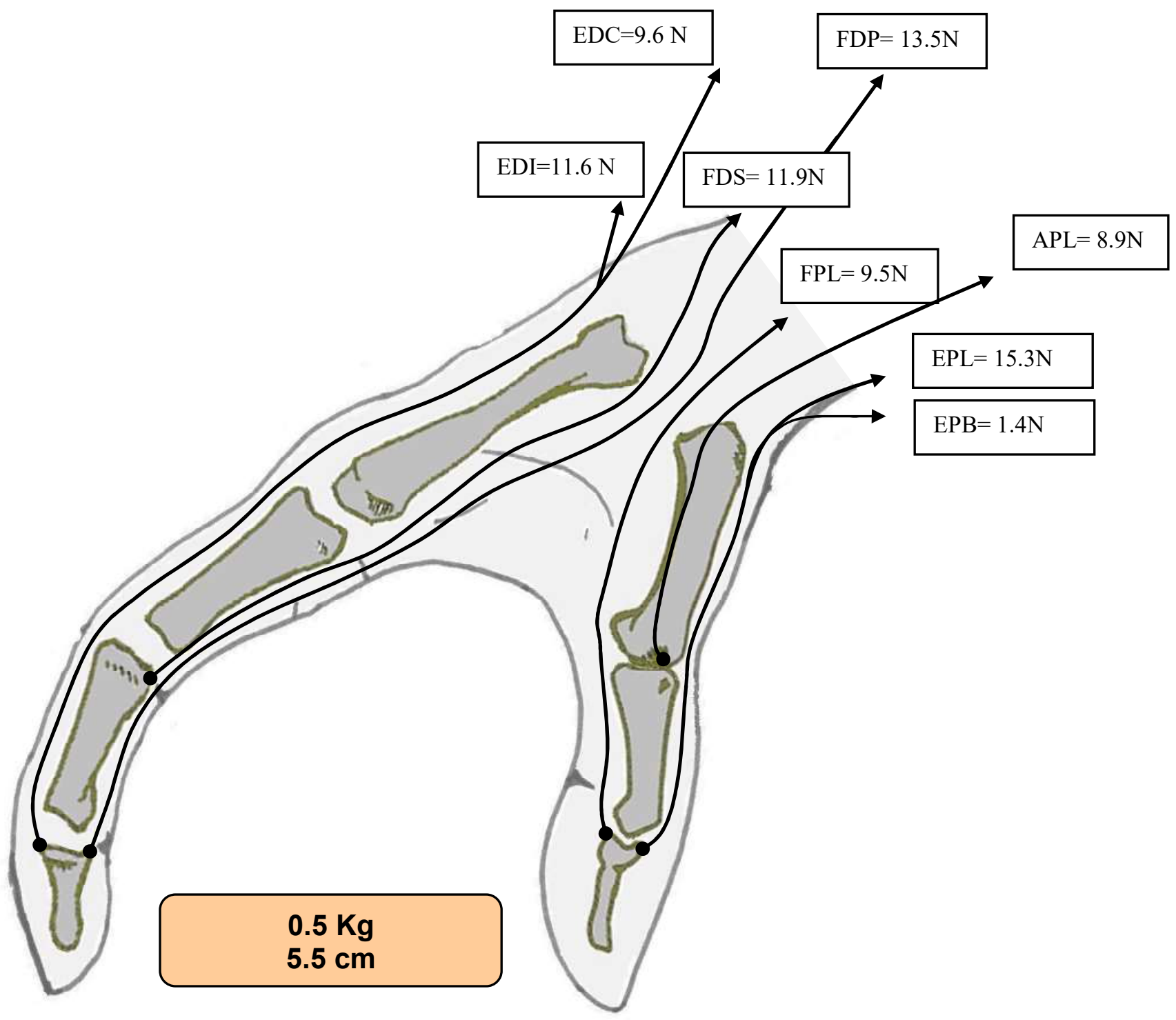


Figure 3:

447

448

449

450

451

452

453

454

455

456

457

458

459

460

$46^{1}$

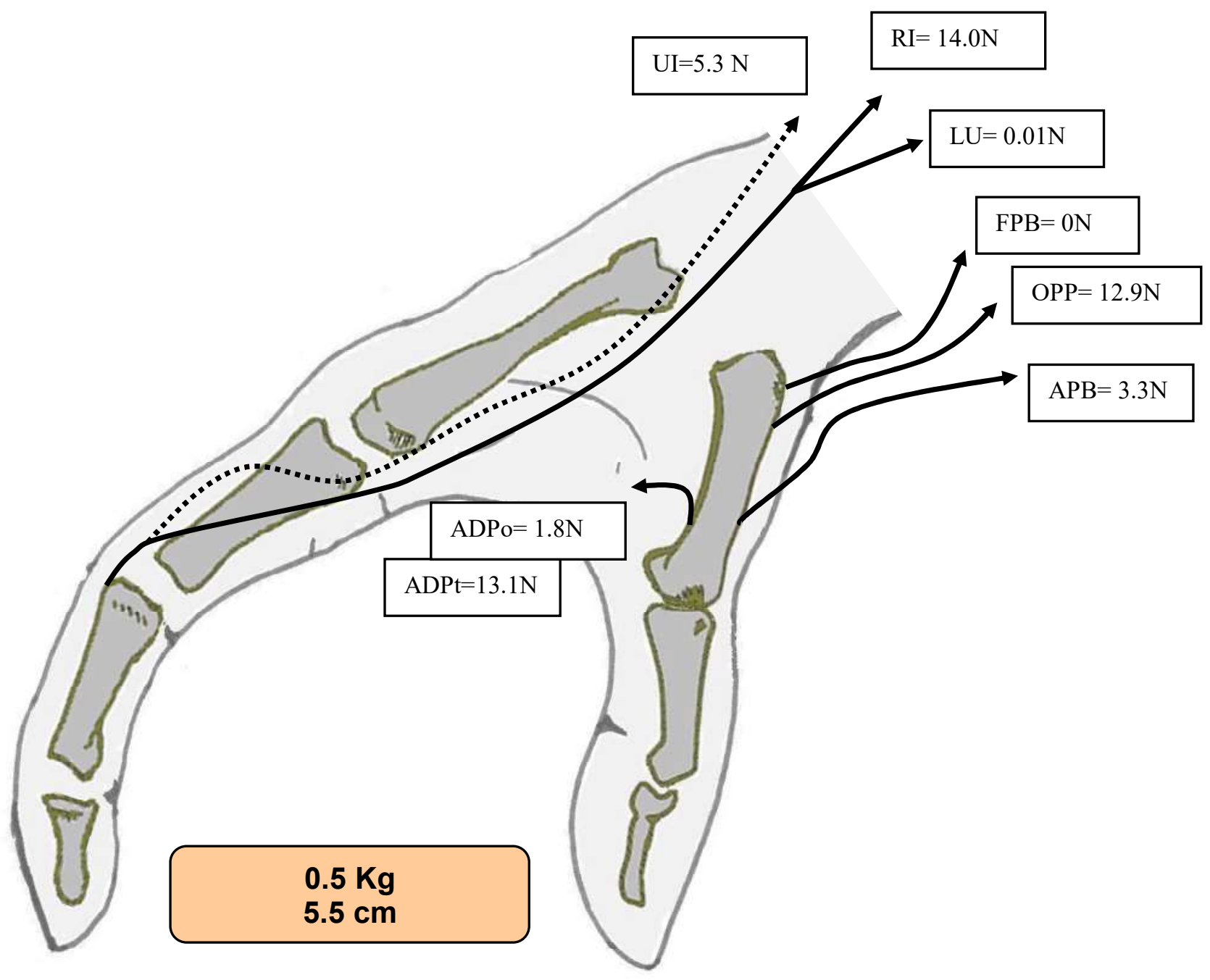

462

463

464 
Figure 4:

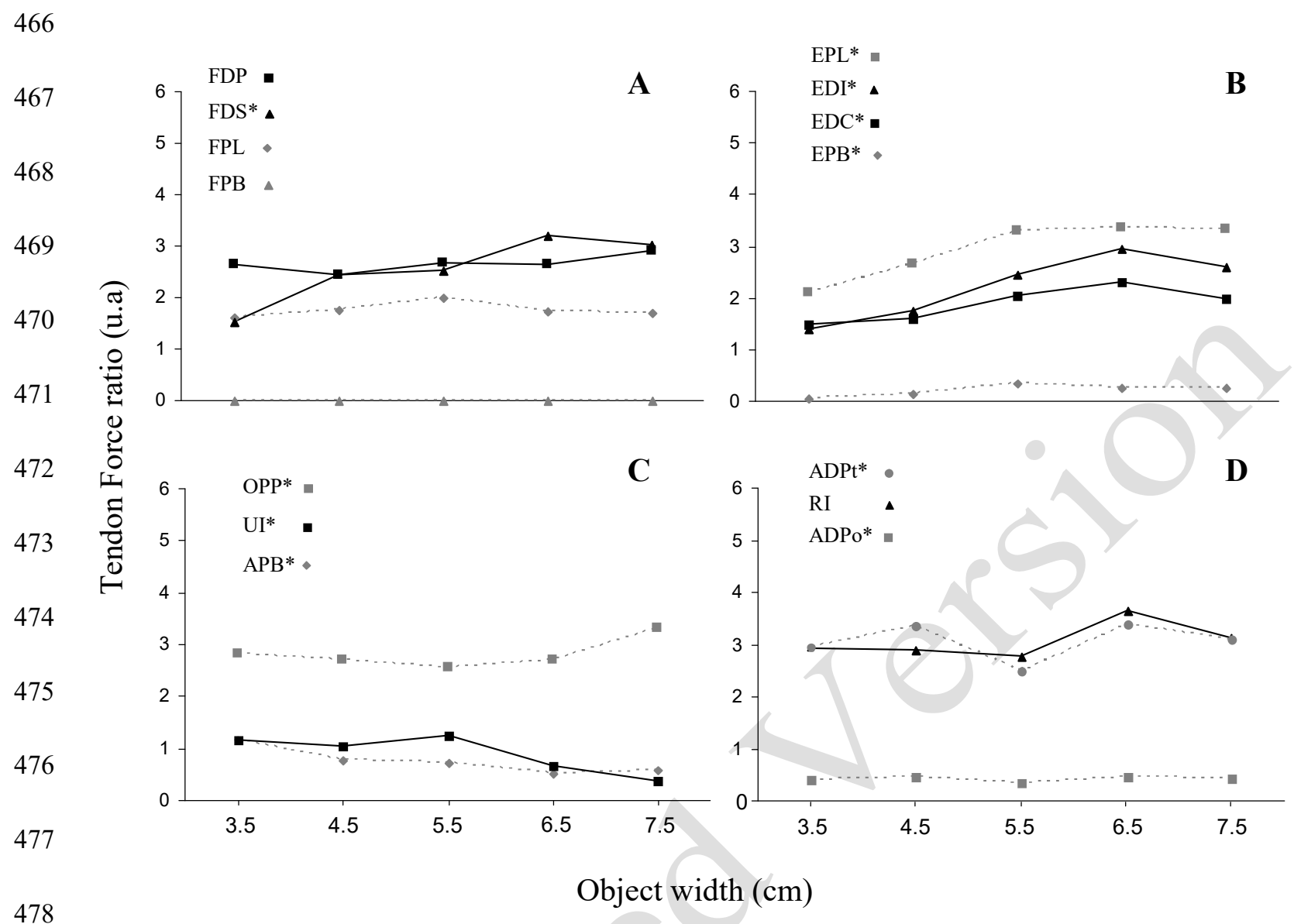


Figure 5:

480
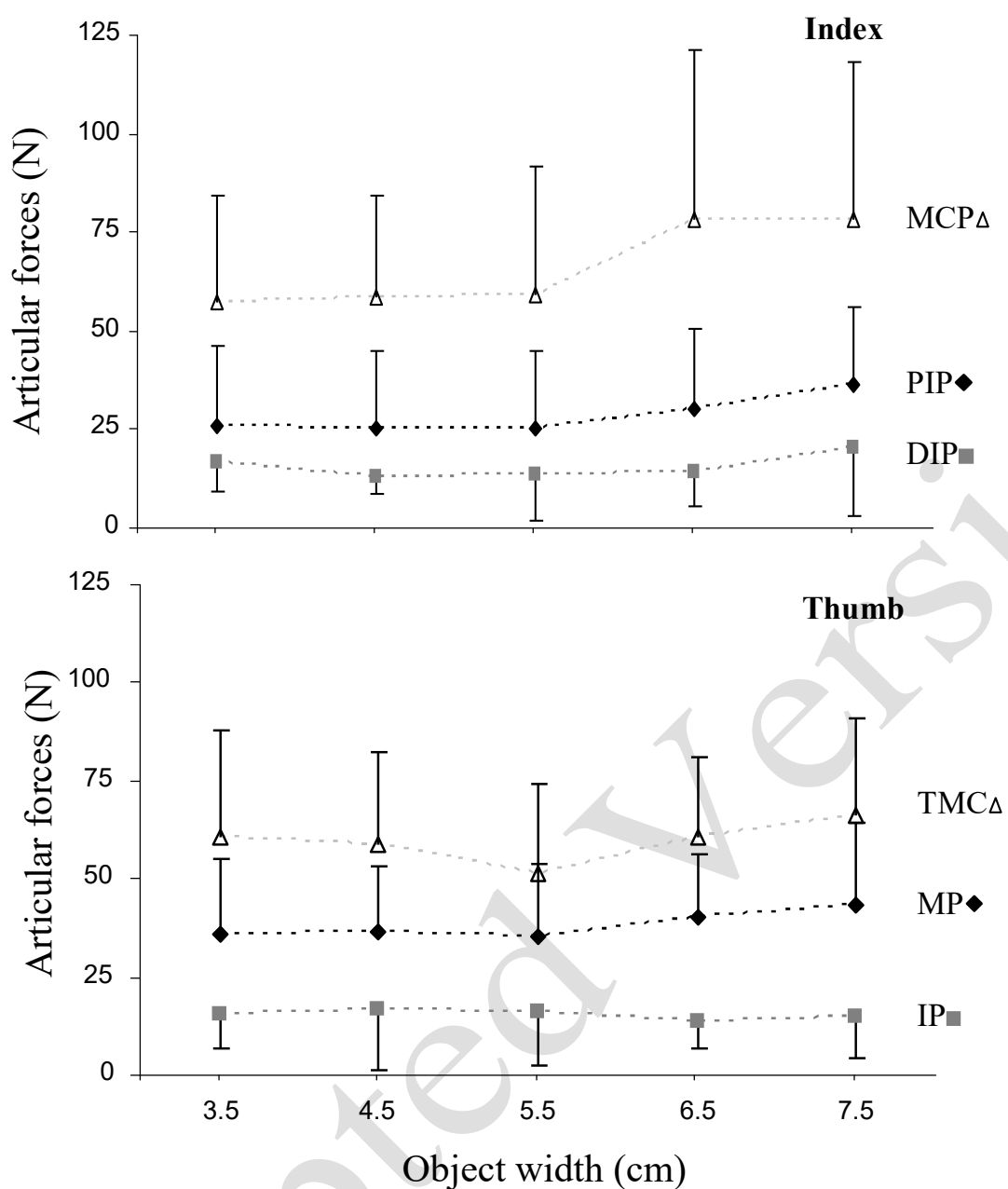